Kong. Res. J. 2(1) : 125-127, 2015

ISSN 2349-2694

Kongunadu Arts and Science College, Coimbatore.

\title{
CHARACTERIZATION OF ANTIFUNGAL ACTIVITY OF THE VARIOUS ACTIVE CONSTITUENTS OF COLEUS FORSKOHLII
}

\author{
Ranjith Santhosh Kumar, D.S.* and B. Lakshman Kumar \\ Department of Biotechnology, Kongunadu Arts\& Science College, Coimbatore. \\ *Email: ranjith.deva87@gmail.com
}

\begin{abstract}
The aim of this study was to evaluate the anti-fungal effect of different solvent extracts of Coleus forskohlii which contains minor diterpenoids, deacetylforskohlin, 9-deoxyforskohlin, 1,9-deoxyforskohlin, 1,9-dideoxy-7-deacetylforskohlin, and four other diterpenoids, along with alkaloids and volatile oils. The solvent extracts included both polarized and non-polarized solvents like methanol, ethylacetate, benzene, chloroform, water, hexane, acetone and ethanol. As a result of this study, the antifungal effect was best observed with the methanolic extract against Candida albicans in comparison to the other extracts. This study also revealed that apart from forskohlin, which was confirmed by Thin Layer Chromatography, other compounds that were extracted with methanol are required to produce the antifungal effect and can be taken forward to pharmaceutical preparations.
\end{abstract}

Keywords: Antifungal, active constituents, Coleus forskohlii.

\section{INTRODUCTION}

Coleus forskohlii also known as Plectranthus barbatus (Lamiaceae) is one of the most significant potential medicinal crops of the future, as its pharmacopoeial properties have been discovered only recently. The tuberous roots have been identified as a rich drug for glaucoma, congestive, cardiomyopathy, asthma and certain cancers. Forskolin primary mode of action is to increase cyclic adenosine monophosphate (cAMP) and cAMP mediated functions, via activation of the enzyme adenylatecyclase (Metzger,et al 1981).Forskolin has been shown to increase cAMP formation in all eukaryotic cells except sperm, without hormonal activation of adenylatecyclase (Seamon, et al1981). Forskolin's potentiation of cAMP in turn inhibits basophil, mast cell degranulation and histamine release (Marone, et al1986); lowers blood pressure (Dubey,et al 1981), intraocular pressure inhibits platelet aggregation (Agarwal, 1982), promotes vasodilation (Wysham et al1986), bronchodilation (Lichey,et al1984), thyroid hormone secretion (Roger, 1987) and stimulates lipolysis in fat cells (Okuda,1992).

Forskolin has been shown to enhance lipolysis, regulated by cAMP, also inhibits fat storage. Forskolin is responsible for virtually all pharmacological activities attributed to Coleus forskohlii and the extracts of this constituent have been used in nearly all existing studies though other plant constituents, such as volatile oils and other diterpenoids and coleonols, contribute to the pharmacological activity and adsorption of forskolin.

Candida albicans causes Candidiasis which is an increasingly important disease that has a worldwide distribution due to the fact that it is a frequent opportunistic pathogen in patients. It is a fungus normally present on the skin and in mucous membrane such as vagina, mouth or rectum. The immune system keeps Candida proliferation under control, but when immune responses are weakened, Candida growth can precede unhindered. The uncontrolled growth of Candida is called Candida over growth. Solid media is generally employed for fungal culture, as the broths are not usually recommended except for fungal blood cultures were bi-phasic medium is used. The media commonly employed is Emmon's modification of Sabouraud dextrose agar. The media may be supplemented with antibiotics, such as gentamicin and chloramphenicol to minimize bacterial contamination and cyclohexamide to inhibit saprobic fungi (Jagdishchander, 1999).

The study of Senff et al., (1990) showed the presence of Candida albicans in samples with scalp psoriasis or seborrhoic dermatitis of the scalp (psoriasis capillitii). Yeasts were found in half of the tongue cultures and in 1/8th of the scalp scales. Further proof that Candida is one of the causes of psoriasis was seen in the study analyzed in more than 40,000 patients which showed that patients with atopic dermatitis and psoriasis had elevated levels of Candida in the intestines (Henseler et al., 
1997).The analysis showed that Candida colonization in the digestive tract can be one of the causes of psoriasis.

\section{MATERIALS AND METHODS}

\subsection{Collection of raw drugs}

The plants of Coleus forskohlii was collected randomly from Tamil Nadu Agricultural University (TNAU) Coimbatore, Coimbatore and Salem regions. The taxonomic identities of these plants were confirmed at Botanical survey of India and the voucher specimen numbers of the plants were preserved. Fresh plant material was washed under running tap water, air dried and then homogenized to fine powder and stored in airtight bottles.

\subsection{Extraction procedure}

$3 \mathrm{~g}$ of air-dried powder was extracted in water and all the solvents in Bio Sox apparatus and then kept on a rotary shaker at $190-220 \mathrm{rpm}$ for $2 \mathrm{~h}$. It was then filtered through 8 layers of muslin cloth and centrifuged at $5000 \mathrm{~g}$ for $10 \mathrm{~min}$. The supernatant was collected. This procedure was repeated twice. The supernatant collected was pooled together and concentrated by evaporating in a vacuum drier to make the final volume one fourth of the original volume and stored at room temperature in airtight bottles.

\subsection{Anti-fungal activity}

\subsubsection{Fungal Strains}

In vitro antimicrobial activity was examined for aqueous and solvent extracts of Coleus forskohlii. The culture of Candida albicans was obtained from the PSG Institute of Medical Sciences and Research, Coimbatore (ATCC11778) and maintained at $4^{\circ} \mathrm{C}$ on nutrient agar slants.

The nutrient broth and nutrient agar were prepared as per the standard composition.

\subsubsection{Method}

Nutrient broth was prepared as per the standard composition and consisted of peptone 5 gm, $\mathrm{NaCl} 5 \mathrm{gm}$, Beef extract $3 \mathrm{gm}$, yeast extract $2 \mathrm{gm}$, pH 6.8 per 1 litre. Nutrient Agar- Peptone $5 \mathrm{gm}, \mathrm{NaCl}$ $5 \mathrm{gm}$, Beef extract $3 \mathrm{gm}$, Yeast extract $2 \mathrm{gm}$, Agar 20 gm, pH6.8 per 1 litre. Nutrient broth prepared for each culture and was sterilized in an autoclave at 1210C for 15 minutes. After sterilization the drug, different concentrations of the extracts were added. Further the cultures Candida sps. was inoculated in the tubes and subcultured weekly. Control sample was prepared along with culture in the absence of the drug. Only medium served as blank.

\subsubsection{Media preparation and antifungal activity}

The antimicrobial assay was performed by agar well diffusion method for all the extracts. The molten Mueller Hinton agar was inoculated with $100 \mu \mathrm{l}$ of the inoculum $\left(1 \times 10^{8} \mathrm{cfu} / \mathrm{ml}\right)$ and poured into the Petri plate (Hi-media). For agar well diffusion method, a well was prepared in the plates with the help of a cork-borer $(0.85 \mathrm{~cm})$. Different concentrations of the test compound were introduced into the well. The plates were incubated overnight at $37^{\circ} \mathrm{C}$. Microbial growth was determined by measuring the diameter of zone of inhibition. Controls were maintained where pure solvents were used instead of the extract. The result was obtained by measuring the zone diameter. The experiment was done in triplicate and the mean values recorded.

\section{RESULTS AND DISCUSSION}

In the present study the different solvent extracts of Coleus forskohlii (methanol, ethyl acetate, benzene, chloroform, water, hexane, ethanol and acetone) at different concentrations $75 \mu \mathrm{g}$ and $150 \mu \mathrm{g}$ was tested for its antimicrobial property against the growth of Candida albicans. The zone of inhibition observed after $72 \mathrm{hrs}$ showed potent activity at $150 \mu \mathrm{g}$ of the methanol extract $(20 \mathrm{~mm})$ and benzene extract $(13 \mathrm{~mm})$ and water $(12 \mathrm{~mm})$, whereas with chloroform it was $6 \mathrm{~mm}$ and other solvents like acetone, ethylacetate, ethanol, hexane did not reveal any zone of inhibition(Table 1 and Figure 1).

The anti microbial activity of plant oils and extracts has been recognized for many years and the significance of the microbial activity of oils and extracts of plants against Candida albicans, Enterococcus faecalis and E. coli has been reported. The oil of lemon grass, oregano and bay leaves were observed to inhibit the growth of most microorganisms (Naveens, 2008). The plant extracts, as methanolic extracts of Peltophorum pterocarpum and Punica granatum exhibited highest antifungal activity against Candida albicans (Duraipanidyan et al., 2006).

The above results imply that the compounds from Coleus forskohlii that was extracted into methanol had the property to inhibit the growth of Candida albicans compared to the other solvents extracts. There was no antagonistic effect on Candida albicans by the Coleus forskohlii extracted in chloroform, petroleum ether and other solvents. However, maximum inhibition was observed with the reference drug clopidogriel, where as DMSO, used as vehicle control showed no inhibitory effect. The studies of screening results show a correlation with the antibiotic uses of the plant and the active 
compounds present in extracts that may be responsible for the antimicrobial activity.

\section{CONCLUSION}

In the present study, methanolic Coleus forskohlii extracts exhibited high level of antifungal activity against Candida albicans.They have an excellent safety profile and are generally without toxicity or side effects at the recommended dosage. In recent years, secondary plant metabolites (phytochemicals), previously with unknown pharmacological activities, have been extensively investigated as a source of medicinal agents. Thus, it is anticipated that phytochemicals with adequate efficacy can be used for the treatment and prevention of various ailments. The potential for developing therapeutic drugs from higher plants appears rewarding as it will lead to the development of a phytomedicine to act against safely in humans. Plant-based drugs have enormous therapeutic potential as they can serve the purpose with lesser side effects that are often associated with synthetic drugs. Continued further exploration of plantderived products is needed today.

Further research is necessary to determine the identity of the compounds from within these plants and also to determine their full spectrum of efficacy. However, the present study of in vitro evaluation of Coleus forskohlii forms a primary platform for further phytochemical and pharmacological studies.

\section{Table 1}

\begin{tabular}{ccc}
\hline S.No & $\begin{array}{c}\text { Forshkolin } \\
\text { extracts }\end{array}$ & $\begin{array}{c}\text { Zone of inhibition } \\
\text { in ( mm) }\end{array}$ \\
\hline 1 & Methanol & 20 \\
2 & Ethyl acetate & - \\
3 & Benzene & 13 \\
4 & Chloroform & - \\
5 & Water & 12 \\
6 & Hexane & - \\
\hline
\end{tabular}

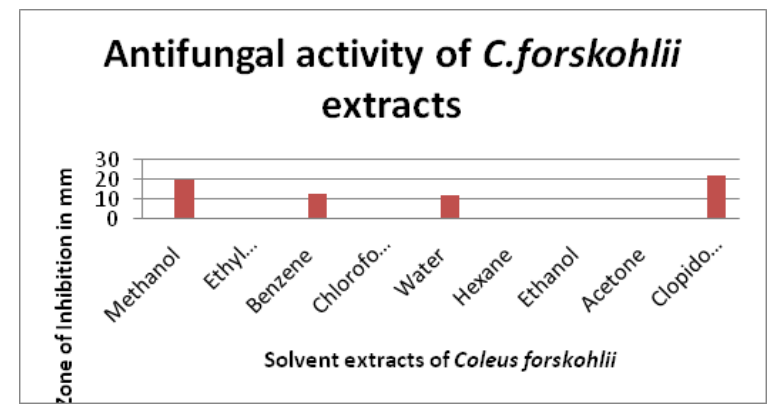

Figure 1

\section{REFERENCES}

Agarwal, K.C. and R.E. Jr. Parks, (1982). Synergistic inhibition of platelet aggregation by forskolin plus PGE1 or 2-fluoroadenosine: effects of 2',5'dideoxyadenosine and 5'-methylthioadenosine. Biochem. Pharmacol. 31: 3713-3716

Buslau, M., I. Menzel and H. Holzmann, (1990). Fungal flora of human faeces in psoriasis and atopic dermatitis. Mycoses 33: 90-94

Dubey, M.P., R.C. Srimal, S. Nityanand and B.N. Dhawan, (1981). Pharmacological studies on coleonol, a hypotensive diterpene from Coleus forskohlii. J. Ethnopharmacol. 3: 1-13.

Marone, G., M. Columbo and M. Triggiani, (1986). Forskolin inhibits the release of histamine from human basophils and mast cells. Agents Actions 18: 96-99.

Metzger, H. and E. Lindner, (1981). The positive inotropic-acting forskolin, a potent adenylate cyclase activator. Arzneimittelforschung 31: 1248-1250.

Naveena, B.M., M. Muthukumar, A.R. Sen, Y. Babji and T.R.K. Murthy, (2006). Improvement of shelf-life of buffalo meat using lactic acid, clove oil and vitamin $\mathrm{C}$ during retail display. Meat Sci. 74: 409-415.

Okuda, H., C. Morimoto and T. Tsujita, (1992). Relationship between cyclic AMP production and lipolysis induced by forskolin in rat fat cells. J. Lipid Res. 33: 225-231

Roger, P.P., P. Servais and J.E. Dumont, (1987). Regulation of dog thyroid epithelial cell cycle by forskolin, and adenylate cyclase activator. Exp. Cell Res. 172: 282-292.

Seamon, K.B., W. Padgett and J.W. Daly, (1981). Forskolin: unique diterpene activator of adenylate cyclase inmembranes and intact cells. Proc. Natl. Acad. Sci. USA 78: 3363-3367

Senff, H., C. Bothe, J. Busacker and D. Reinel, (1990). Studies on the yeast flora in patients suffering from psoriasis capillitii or seborrhoic dermatitis of the scalp. Mycoses 33: 29-32.

Srinivasan, S., M. Anitha, S. Mwangi and R.O. Heuckeroth, (2005). Enteric neuroblasts require the phosphatidylinositol 3-

kinase/Akt/Forkhead pathway for GDNFstimulated survival. Mol. Cell. Neurosci. 29: 107119

Veeramuthu Duraipandiyan, Muniyappan Ayyanar and Savarimuthu Ignachimuthu, (2006). Complemen. Alter. Med. 1-7.

Wysham, D.G., A.F. Brotherton and D.D. Heistad, (1986). Effects of forskolin on cerebral blood flow: implications for a role of adenylate cyclase. Stroke 17: 1299-1303. 